\title{
Pre Analytical Phase : The Seismic Zone of Clinical Laboratory Testing
}

\author{
Rateesh Sareen*, Menka Kapil and G N Guta \\ Department of Pathology \& Transfusion Medicine, Santokba Durlabhji Hospital(India)
}

\begin{abstract}
The total testing process is divided into preanalytical, analytical and post analytical phase, with most of the errors has been suggested to occur in pre analytical phase. Pre analytical phase is the seismic zone in terms of errors as this phase has processes that are generally held outside the clinical laboratory and not under the control of the laboratory management. Therefore, active monitoring and control of all possible defects that are caused by non -laboratory personnel is essential for incorporating actions outside the laboratory in the laboratory quality assurance plan. Measures to reduce preanalytical errors include- continuous education \& training, written policies, implementation of standard operating procedures, establishment of quality indicators and communication. It is the involvement of the entire health care team as a single unit that can bring improvement in total testing process and specifically pre analytical phase.
\end{abstract}

Keywords: Preanalytical Phase, Quality Indicators

\section{Introduction}

The creditability of laboratory test results rely heavily on each and every step in total testing process performed correctly at the right time, in right manner by right personnel. A quality laboratory report ensures timely medical decision making. Total testing process encompasses nine critical steps: ordering, collection, identification, transportation, separation or preparation, analysis, reporting and action ${ }^{[1]}$. Every parameter has three phases of testing- Pre analytical, analytical and post analytical phase. There have been significant improvement in the quality of laboratory test results with improvement in reliability and standardization of analytical techniques, reagents and instrumentation. [2] The ISO 15189:2007 standard for laboratory accreditation defines the preanalytical phase as 'steps starting in chronological order from clinician's request and include examination of request form, collection of sample and the transport to the laboratory or within the laboratory culminating in analytical phase of testing. ${ }^{[3]}$ The preanalytical phase accounts for nearly $70 \%$ of the errors in laboratory testing. ${ }^{[4]}$ The preanalytical phase beyond doubt is the most error prone phase but recent data from literature suggests that the errors occurring in the preanalytical phase are mostly related to procedures performed outside the laboratory by health care personnel who are not under direct control of laboratory. ${ }^{[5]}$ For example, we are all aware of effect of hemolysis on serum Potassium concentration. The sample collection by nursing staff in inpatients inwards, ICU and clinical areas is more prone to errors than by sample collection done by laboratory personnel or phlebotomist. Again the nursing staff is not directly under the control of laboratory. The laboratory personnel can train, evaluate competency of nursing staff for sample collection but cannot directly supervise them as both have different domains of working. However, the laboratory relies on the sample collection technique by nursing staff and analysis of samples is done with reporting of tests. Therefore it is imperative that quality improvement must take in to account both the 'classical' preanalytical phase and the procedures that are part of preanalytical phase included in the 'pre-preanalytical phase', which are performed neither in the laboratory nor at least in part, under the direct control of the laboratory. ${ }^{[6]}$

\section{Discussion}

The preanalytical phase is riddled with lax attitude about filling of requisition to lack of education about ideal phlebotomy procedure. Plebani and Carraro in their papers have emphasized on the importance of pre analytical phase as a major determinant for curtailing errors in laboratory medicine ${ }^{7}$ The lack of external quality assessment for pre analytical phase further complicates the issue as bench marking is not possible for this phase of testing.

The important errors in preanalytical phase are as follows

i) Incomplete \& Inappropriate Laboratory Test Request form: Incomplete request forms are rarely rejected at the laboratory reception as many times the staff at reception are un aware of the significance of missing data. The information may be physician name, patient identifier and requested test. As per literature studies the inappropriate tests may vary from 11 to $70 \%$ for general biochemistry \& hematology, $5 \%$ to 
95\% for urine screening and microbiology and $17.4 \%$ to $55 \%$ for thyroid and cardiac enzymes. ${ }^{[8,9]}$

ii) Patient Identification Inappropriate: It is a suicide for laboratory to report on a wrong sample. This error is the most serious one that can seriously jeopardize patient outcome and give rise to medico legal complication. The use of electronic technologies like barcodes, radiofrequency identification and wrist bands bearing patient identifiers should be a part of system so as to eliminate this error. ${ }^{[10,11]}$

iii) Incorrect Labeling: Labelling of containers should always be done prior to sample collection in the container as post sample collection labeling leads to increased error.

iv) Error in Collection: Inadequate volume of samples, Hemolysed samples, lipemic samples and coagulated samples all lead to preanalytical errors. Hemolysed samples occur when blood is forced through fine needle ,shaking the tubes vigorously and centrifuging the sample specimens before clotting. ${ }^{12}$ Although the introduction of vacuum tubes along with the closed system of blood collection has made blood collection efficient and easy but the lack of training of staff engaged in phlebotomy is impediment for expediting sample collection and transport. Red top vacutainers should not be shaken after sample has been collected and vacutainers for plasma should be gently inverted a few times so that there is complete mixing of blood with anticoagulant. Hemolysis causes extravasation of intracellular contents into plasma, leading to false high values of potassium, aspartate amino transferase (AST) and Lactate dehydrogenase (LDH).

v) Transport Delay: In case of non optimized transport of specimens at the prescribed temperature is done it may lead to errors. There should be recording of transport time, conditions like humidity \& temperature parameters and continuously monitoring of the above for optimal performance by laboratory. ${ }^{[13]}$

vi) Error in Specimen Preparation: Specimens are potentially hazardous material and should be handled with care and safely not only for correct analysis but also for maintain safety of laboratory staff.

The laboratory should adopt policy for rejection of samples that are not for testing, it is difficult preposition but at the same time one must bear in mind that a poor sample will give poor results.

Most of the errors are likely to result in minimal harm to the patient because either they remain undetected or are judged as clinically insignificant and are further ignored by referring physician but this does not mean that they can be overlooked. ${ }^{14}$ A plausible solution for reducing pre analytical errors is by the use of quality indicators covering all steps in the pre analytical phase. Quality indicators which are the objective measure to evaluate all critical care domains as defined by Institute of Medicine (IOM) are evidence based and can be implemented in consistent, comparable manner across all settings. ${ }^{15}$ It is therefore necessary that there should be consistent and systematic data collection, analysis using set of indicators that address all stages of total testing process and targeted focus on areas which have impact on patient care and health outcome.

It was in 2008 under the aegis of The international federation of clinical chemistry and laboratory medicine (IFCC) in 2008 a working group named 'Laboratory errors and patient safety' (WG-LEPS) evaluated relevant quality indicators to address all stages of total testing process. ${ }^{[15]}$ The model suggested by WG-LEPS complied with the requirements of the standard UNI 11097 as per which a quality indicator is, " the information, qualitative or quantitative that is able to evaluate its change during the time and to verify the defined quality goals in order to take correct decisions and choices'. ${ }^{[16]}$

The 16 quality indicators as developed by the IFCC WGLEPS for the preanalytical phase are shown in table -1

The development of quality indicators in laboratory is the fundamental step that provides evidence of quality process in TTP but mere collection and monitoring of data does not result in quality improvement unless efforts are made to achieve consensus on the preparation, adoption and monitoring of effective standard operating procedures in preanalytical phase of laboratory testing. ${ }^{[5,17]}$

\section{Conclusion}

Quality assurance is a never ending journey. The interrelated steps that can prevent preanalytical errors are: a) short \& clear written procedures, b) improvement in health care personnel continuous education \& training, c) implementation of standard operating procedures, d) establishing quality indicators and e) improvement in communication between health care professionals and individuals departments. The laboratory staff should not only know the procedures and the consequences of non compliance \& corrective action but they must also know about the errors and their effect on the sample, test result and finally the patient. There should be ongoing training for these employees and annual competence assessment. The testing areas involving non laboratory personnel should have excellent communication with the laboratory. Adequate and effective training of personnel on processes 
Table 1: Quality indicator (QI) in Preanalytical phase.

\begin{tabular}{|c|c|c|}
\hline Ql-1 & Appropriateness of test request & No of Requests with clinical question (\%) \\
\hline QI-2 & Appropriateness of test request & No of appropriate tests with clinical question (\%) \\
\hline QI-3 & Examination requisition & No of requests without physician identification (\%) \\
\hline QI-4 & Examination requisition & No of unintelligible request (\%) \\
\hline QI-5 & Identification & No of requests with erroneous patient identification (\%) \\
\hline QI-6 & Identification & No of requests with erroneous physician's identification (\%) \\
\hline QI-7 & Test request & No of requests with errors concerning test input (\%) \\
\hline QI-8 & Samples & No of samples lost/ not received (\%) \\
\hline QI-9 & Samples & No of samples in wrong containers (\%) \\
\hline QI-10 & Samples & No of samples hemolysed (\%) \\
\hline QI-11 & Samples & No of samples in clotted (\%) \\
\hline Q।-12 & Samples & No of samples with insufficient volume (\%) \\
\hline Q।-13 & Samples & No of samples with inadequate sample- anticoagulant ratio (\%) \\
\hline Q।-14 & Samples & No of samples damaged in transport (\%) \\
\hline QI-15 & Samples & No of samples improperly labeled (\%) \\
\hline Q।-16 & Samples & No of samples improperly stored (\%) \\
\hline
\end{tabular}

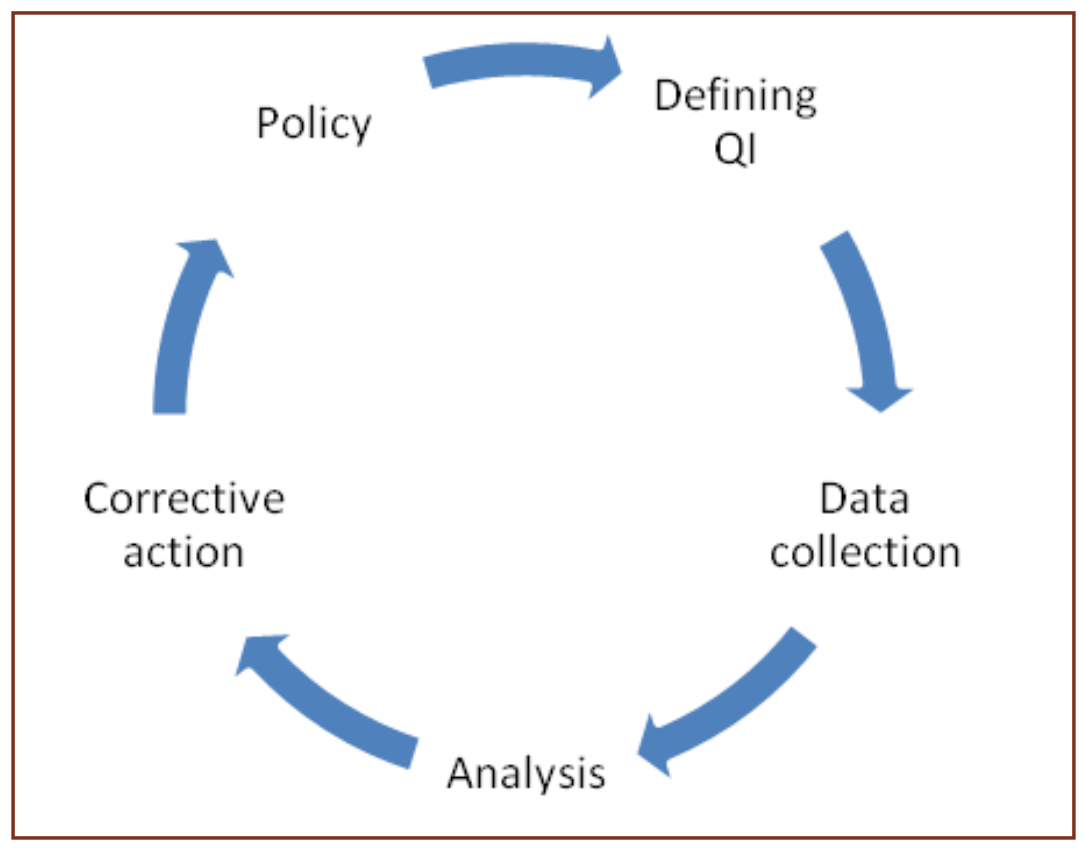

Fig. 1: QI (Quality Indicator) as a tool in laboratory improvement. 
and procedures should be given top priority in institutes, hospitals and laboratories involving entire health care system so that we can witness improvement in quality of overall testing process.

\section{Reference}

1. Lundberg GD. Acting on significant laboratory results. JAMA $1981 ; 245: 1762-3$

2. Plebani M. Errors in clinical laboratories or errors in laboratory medicine? Clin Chem Lab Med 2006; 44: 750-9.

3. International Organization for Standardization. ISO 15189:2007: Medical laboratories: particular requirements for quality and competence. Geneva, Switzerland: ISO; 2007.

4. Lippi G, Chance JJ, Church S, Dazzi P, Fontana R, Giavarina D, et al. Preanalytical quality improvement: from dream to reality. Clin Chem Lab Med 2011; 49: 1113-26.

5. Carraro P, Zago T, Plebani M. Exploring the initial steps of the testing process: frequency and nature of prepreanalytic errors. Clin Chem 2012; 58:638-42.

6. Plebani M. Exploring the iceberg of errors in laboratory medicine. Clin Chim Acta 2009; 404:16-23.

7. Bonini P, Plebani M, Ceriotti F, et al. Errors in laboratory medicine. Clin Chem. 2002; 48:691-698.

8. Silverstein, MD. An approach to medical errors and patient safety in laboratory services. In: A white paper. Atlanta: The Quality Institute Meeting; 2003.
9. Kirchner MJ, Funes VA, Adzet CB. Quality indicators and specifications for key processes in clinical laboratories: a preliminary experience. Clin Chem Lab Med 2007; 45:672-7.

10. D Dzik WH. New technology for transfusion safety, Br J Haematol 2007; 36:181-90.

11. Lau FY, Wong R Chui CH. Improvement in transfusion safety using a specially designed transfusion wristband. Transfus Med 2000; 10:121-4.

12. Lippi G, Bassi A, Brocco G. Preanalytic error tracking in a laboratory medicine department: results of a 1-year experience. Clin Chem 2006; 52:1442-4.

13. Astion ML, Shojania KG,Hamill TR. Classifying laboratory incident reports to identify problems that jeopardize patient safety. Am J Clin Pathol2003; 120:1826.

14. Plebani M, Sciacovelli L, Aita A, Padoan A, Chiozza ML. Quality indicators to detect pre-analytical errors in laboratory testing. Clinica Chimica Acta. 2014; 432: 44-48.

15. Institute of Medicine Committee on Quality of Health Care in America. To Err Is Human: Building a Safer Health System. Washington, DC: National Academies Press; 2000.

16. Sciacovelli L, Plebani M. The IFCC Working Group on laboratory errors and patient safety. Clin Chim Acta 2009;404:79-85

17. Sciacovelli L, Sonntag O, Padoan A, Zambon CF, Carraro $\mathrm{P}$, Plebani M. Monitoring quality indicators in laboratory medicine does not automatically result in quality improvement. Clin Chem Lab Med 2011; 50: 463-9.

*Corresponding author:

Dr Rateesh Sareen, D.N.B Pathology Department of Pathology \& Transfusion Medicine, Santokba Durlabhji Hospital(India).

Phone: +91 01412566251

Email: drrateeshsareen@yahoo.co.in.

Financial or other Competing Interests: None.

Annals of Advanced Medical Science, Vol. 2; Issue 2, April -June, 2018 